\title{
Management of Concurrent DDA and EBA eBook Programs
}

\section{or, Adventures in Deduplication for Acquisitions}

\author{
by Christina Torbert
}

Libraries currently have a variety of methods by which they can acquire ebooks. At the University of Mississippi-a medium-sized research institution with a large undergraduate population-we have mostly purchased or subscribed to content at ProQuest Ebook Central (also previously known as Ebrary), with some smaller number of titles on the EBSCO platform. Early ventures (before I was over acquisitions) included the purchase of a NetLibrary collection (now held by EBSCO) and a subject-specific subscription collection from ProQuest. About ten years ago (still before I was in charge), the library began to purchase ebooks through a demand-driven-acquisitions (DDA) program. This program uses selection profiles already established in GOBI (a book-purchasing platform owned by EBSCO) by the liaisons to produce a large collection of ebooks from multiple publishers that might be useful to our patrons. The library makes those titles discoverable and, as any individual title is used in a substantial way, a perpetual purchase is triggered and the library is invoiced for the purchase. Eventually, unpurchased titles are removed from the catalog and newer titles are added. The library receives possible DDA titles on both the PQ and EBSCO platforms with no substantial restrictions on publishers included. Because we limit the timeframe of the DDA program (most recent two years), there is no overlap with our subscription package.

The library has also participated in a few small, evidence-based acquisitions (EBA) programs through the years. An EBA works similarly to DDA, but the titles are all restricted to a single publisher or platform and all the purchases are made by library selection at the end of a restricted timeline. The price for the program is decided and paid at the beginning, related to the amount of content that will be made available for use. Title-level records are made available in the catalog and, at the end of the timeframe (usually a year), liaisons may make their selections based on the usage of titles-i.e., evidence of need.

The library has participated in limited EBA programs over the years, but during this past year we had three separate programs running at different times: Oxford University Press for music, literature, history, and philosophy content from 2018 and 2019; Cambridge University Press for all music content; and Taylor \& Francis content from 2018 forward for all subject areas. While the materials included in the Cambridge EBA were not likely to overlap with titles in the comprehensive DDA, the titles in the OUP and the T\&F programs were probably going to be duplicates of titles available on the platforms covered by our DDA.

Due to the timing of the programs, I had to take two different paths to reducing the possibility of purchasing the same content on two different platforms. Prior to starting the OUP EBA, my colleague, the Collection Strategist, did an analysis of the titles previously purchased through DDA from OUP, and the library's purchases from OUP were primarily in the subject areas participating in the EBA. After consulting with the subject liaisons, we excluded OUP titles from the DDA program for the year and asked the liaisons to not order OUP content for the year unless requested by a patron. We were not able to insert indications in GOBI to remind liaisons of this EBA program, so there was still a chance that duplicate content might be purchased via individual purchases. This

Christina Torbert is the Head of Continuing Resources and Acquisitions and the Liaison to the Deptartment of Philosophy and Religion at the University of Mississippi. 
exclusion of a single publisher meant a reload of the DDA records to remove the excluded titles. OUP provided catalog records for the titles included in the EBA but, as I was double-checking access, I discovered that they had also included records for open access titles, which was confusing.

When we went to set up the T\&F EBA just a couple months later, GOBI had established a workflow that allowed us to run our EBA program through them. They worked with T\&F to learn which titles would be included in the EBA, and GOBI passed that information on to the platforms handling the DDA content so the T\&F titles were excluded automatically. GOBI was also able to place a note on the titles included in EBA in their ordering interface so that liaisons would know not to order those titles. Unfortunately, GOBI could not help us remove the T\&F DDA records from the online catalog, and I had to run lists in the catalog to try to suppress as many of those records that still pointed to the aggregator sites as possible, hoping that only the records linking to T\&F would be exposed for discovery. T\&F also supplied catalog records for the titles included and, because 2020 content was included, they continued to send update files.

Let me back up and describe how our library handles ebooks in the online catalog. We only add records for titles that have been purchased or that might be purchased. We do not add separate records for ebooks available through subscription, through databases, or through open access. We track those collections exclusively through our discovery layer and the management tools found there. Because our discovery layer is owned by the same company as the aggregator through which we subscribe and which we have as our primary DDA platform, the vendor is able to auto-update our title-level holdings for us for the titles on their platform into the knowledgebase, but all other platforms have to be manually updated. Therefore, for most ebooks in the discovery knowledgebase, we try to work at collection-level tracking rather than at title-level. This practice is slowly changing as we work to get more of the purchased ebooks into the knowledgebase. What indicates a purchased title in the catalog is the attached order and payment record. I mentioned previously that, as the years of coverage roll forward for DDA, we remove unpurchased bib records from the catalog. The cleanest way to accomplish this refresh is to gather up and delete all ebook records without an attached payment record and then reload a full set of records from Gobi. We try to limit this type of reload to once a year. We also use the added author/title fields (710/740) to track which "collection" an individual record belongs to-i.e., YPB DDA, PQ individually purchased, [publisher] evidence-based selection. We add these fields when the bib record is uploaded and use them to find particular collections again later when we need to eliminate unpurchased titles.

For example, as the OUP EBA was ending, we received from OUP a report of usage from the past year. After weeding out the titles we had already purchased (nice to know they got use), the discounted price of the remaining used titles did not come to the amount we had determined to spend. We turned to the titles that had received turnaways during the past year, and we made several selections from that list to bring our spend up to the required level. We added order records to the titles with bib records already in the catalog and made a list of titles for which we still needed bib records. It took a couple tries to get the correct records from OUP but, once all the titles had order records attached, we ran a list of bibs with the correct added collection title (740) and no attached order record. Those bibs were deleted from the catalog. We will eventually send the list of purchased titles to GOBI so they can be notated in the ordering interface, and we will have OUP titles restored to the DDA for future loads.

I continue to have mixed feelings about the effectiveness of EBA programs, especially compared to DDA programs. On the positive side, the library is sure to get quality content when it selects a 
specific publisher and, for a pricey publisher like OUP, the discount makes the costs more affordable. Many of the titles we purchased through EBA would have been outside the price parameters of our DDA programs and so would never have been available for discovery and use. But I'm not sure one year's worth of use is enough to judge on for the amount of money required up front in an EBA program, and we often have to purchase titles that would not have triggered a DDA purchase in order to spend the required amount. Also, working directly with the publisher means the library has to handle more of the administrative burden: deduping the title lists, noting the holdings in the agent's interface, getting the correct MARC records... So far, working through the intermediary service of the book agent GOBI has streamlined this process. I may re-revaluate this assessment when reconciling the T\&F EBA in the fall. 